\title{
Switching controller for efficient IBVS
}

\author{
Guillaume Allibert, Estelle Courtial
}

\begin{abstract}
Image-based visual servoing (IBVS) is now recognized to be an efficient and robust control strategy to guide robots using only visual data. Classical IBVS is commonly based on the Cartesian coordinates of points in the image. Although the convergence of the visual features to the desired ones is generally achieved, classical IBVS can lead to unnecessary displacements of the camera, such as the camera retreat problem in the case of a pure rotation around the optical axis. In contrast, IBVS based on the polar coordinates of points, is well adapted to carry out rotations around the optical axis but less adapted to manage translations. To take advantage of the benefits of each approach, we propose a new strategy for visual servoing based on a switching controller. The controller switches between Cartesian-based and polar-based approaches thanks to a switching signal provided by a decision maker. With the proposed controller, unnecessary 3D displacements are minimized without any 3D reconstruction and visibility constraints can be taken into account. The local stability of the closed-loop switching system is proved. A comparison with classical controllers and advanced controllers is performed by simulations. This comparative study illustrates the effectiveness of the proposed controller in terms of displacement in the image space and in the $3 D$ space.
\end{abstract}

\section{INTRODUCTION}

Visual servoing is a feedback strategy to control the movement of a robot by using only visual information [2], [3]. Classically, two approaches are distinguished: image-based visual servoing (IBVS) and position-based visual servoing (PBVS). In IBVS, an error signal is measured in the image and the control objective consists in decreasing exponentially this signal to zero. Because it does not require 3D reconstruction, the IBVS strategy offers easy implementation and robustness in the face of measurement noise and model uncertainties. On the other hand, there is no control over the camera position. Unnecessary displacements that can lead to the system failure can be performed in the 3D space. Different IBVS approaches have been developed to avoid or reduce the useless displacement in the 3D space. The partitioned approach [6] addressed this problem by decoupling and servoing the $Z$-axis rotational and translational components of the control. Two new image features (an angle and the square root of area) were computed. A potential function was also incorporated to keep the features in the image plane. In [5], Chaumette proposed to use image moments to design a decoupled IBVS. When a combination of moments is well chosen, a satisfactory behavior of the features was obtained in the image plane as well as an adequate trajectory

G. Allibert is with the laboratory I3S-CNRS/UNS, University of Nice Sophia Antipolis, France. Guillaume.Alliberteunice.fr

E. Courtial is with the laboratory PRISME EA 4229, University of Orléans, France. Estelle.Courtial@univ-orleans.fr in the 3D space. To do this, the object and camera planes were parallel at the desired position and the object was a symmetrical object (but not a square object). The epipolar geometry was also used in hybrid methods [8], [11]. Based on the homography between the desired and initial images, these methods required coplanar feature points and an important computational load. An alternate approach [12] proposed different control schemes based on second-order minimization technique to avoid the camera advance/retreat problem. However, the estimation of the depth at the current instant was required.

In PBVS, the camera position is estimated from the visual features extracted from the current image. If the pose is perfectly estimated, zeroing the error in the 3D-space provides a straight trajectory of the camera. There is no control in the image plane, which can result in the target getting out of the camera field of view. Several studies have addressed the complementarity of the two approaches [9],[10]. In [10], a switched system using both IBVS and PBVS was developed. The switching was done when the image error or the pose error was considered too large. However, the approach remained susceptible to local minima and image singularities. In [9], a hybrid control strategy consisting of a local switching control between IBVS and PBVS was developed in order to avoid image singularities, local minima and to take into account visibility constraints. While PBVS makes it possible to deal with local minima and image singularities, a 3D model of the target is required. Furthermore, PBVS is not robust to modeling and image processing errors when the features considered are points. In order to overcome the need of 3D information, the authors in [7] focused on the capabilities of IBVS by using two different parametrizations. The proposed hybrid visual servoing combined both Cartesian and polar image coordinates by stacking the respective interaction matrices. The induced trajectories were no longer straight lines in the Cartesian and polar image plane. The performance was a mean of performances, with no optimality. Furthermore visibility constraints could not be addressed.

In this paper, we propose, not to combine but to switch between Cartesian and polar-based approaches in order to take advantage of the benefits of each approach. The most difficult problem is to determine when the switch should be done. A decision maker provides the switching signal according to the current value of the visual features and criteria. The latter are based on the prediction of the camera displacements up to the desired position. The switching controller, based on simple visual features like points (coplanar or not), minimizes unnecessary displacements of 
the camera without $3 \mathrm{D}$ reconstruction and can take into account visibility constraints.

The paper is organized as follows. Section II recalls the IBVS strategy respectively based on Cartesian coordinates, named $I B V S_{C}$, and on polar coordinates, named $I B V S_{P}$. The advantages and drawbacks of each controller are underlined. In Section III, the new controller is presented: the switching control architecture, the decision maker, the handling of visibility constraints and finally the stability analysis. In Section IV, numerous simulations show the efficiency of the proposed controller by comparing its performances to those obtained with classical controllers $\left(I B V S_{C}\right.$ and $\left.I B V S_{P}\right)$ or with advanced controllers (controller using image moments [5] or using second-order minimization techniques [12]). Conclusions are given in Section V.

\section{IBVS IN CARTESIAN AND POLAR COORDINATES}

The objective of IBVS is to regulate to zero the image error $e$ between the current value of image features $s$ and its desired value $s^{\star}$. The current value of image features is provided by measurements in the image plane which are usually the pixel coordinates of a set of $n$ image points $(n \geq$ $3)$. In the following, theoretical developments are carried out for only one point.

\section{A. In Cartesian coordinates}

Consider $P$ a 3 -D point with coordinates $(X, Y, Z)$ in the camera frame where $Z$ is perpendicular to the image plane. Its projection in the image plane as a 2-D point $s_{c}$ makes it possible to write in the normalized case:

$$
s_{c}=(u, v)=(X / Z, Y / Z)
$$

where $(u, v)$ are the Cartesian coordinates of an image point feature. The time derivative of (1) yields the fundamental relationship of IBVS:

$$
\dot{s}_{c}=L_{s_{c}} \tau
$$

where $L_{s_{c}} \in \mathbb{R}^{2 \times 6}$ is the interaction matrix and $\tau=$ $\left[T_{x}, T_{y}, T_{z}, w_{x}, w_{y}, w_{z}\right] \in \mathbb{R}^{6 \times 1}$ is the spatial velocity of the camera. The interaction matrix based on Cartesian coordinates of one image point feature is given by [2]:

$$
L_{s_{c}}=\left[\begin{array}{cccccc}
-\frac{1}{Z} & 0 & \frac{u}{Z} & u v & -\left(1+u^{2}\right) & v \\
0 & -\frac{1}{Z} & \frac{v}{Z} & 1+v^{2} & -u v & -u
\end{array}\right] .
$$

Note that $L_{S_{c}}$ depends on $Z$ which is the depth of the point relative to the camera frame. This value must be estimated or approximated, and is noted $\hat{Z}$ in the sequel.

An IBVS controller is generally designed such that the image error exponentially decreases:

$$
\dot{e_{c}}=-\lambda e_{c} \text { with } e_{c}=s_{c}-s_{c}^{\star} \text { and } \lambda>0,
$$

where $s_{c}^{\star}$ is the Cartesian coordinates of the desired image point. Combining (2) and (4), the resulting control law is then given by:

$$
\tau=-\lambda \widehat{L_{s_{c}}}+e_{c}
$$

where ${\widehat{L_{S_{c}}}}^{+}$is the pseudo-inverse of the interaction matrix evaluated with the approximated depth $\hat{Z}$.

With this parametrization, the trajectories obtained in the image plane are straight lines when $n=3$ and $Z$ is perfectly known. Due to the particular expression of the interaction matrix (3), the $I B V S_{C}$ controller is well adapted to deal with translational displacements. Indeed, the camera translations along the $X$ and $Y$ axes are invariant to image point variations respectively along the $u$ and $v$ axes as can be seen in the first and second columns of (3). Nevertheless, in the case of pure rotation around the optical axis, the camera movement is composed of a rotational displacement but also of a translational displacement along the optical axis. This phenomenon is referred to as camera retreat [4]. This unnecessary movement is due to the coupling between the third and sixth columns in (3). To sum up, the $I B V S_{C}$ controller is generally efficient but in particular cases, it can generate unnecessary 3D displacements.

\section{B. In polar coordinates}

The coordinates of an image point feature can also be expressed in polar coordinates [3]:

$$
s_{p}=(\rho, \theta)=\left(\sqrt{u^{2}+v^{2}}, \operatorname{atan}\left(\frac{v}{u}\right)\right)=\phi_{c p}\left(s_{c}\right)
$$

where $\rho$ is the radius and $\theta$ is the angle. As previously, the following equations have ensued from the time derivative of (6):

$$
\dot{s}_{p}=L_{s_{p}} \tau
$$

and the feedback control law:

$$
\tau=-\lambda \widehat{L_{s_{p}}}+e_{p}
$$

$L_{s_{p}} \in \mathbb{R}^{2 \times 6}$ is the interaction matrix based on polar coordinates. Its expression for one point is given by:

$$
L_{s p}=\left[\begin{array}{cccccc}
\frac{c}{Z} & \frac{s}{Z} & -\frac{\rho}{Z} & -\left(1+\rho^{2}\right) s & \left(1+\rho^{2}\right) c & 0 \\
-\frac{s}{\rho Z} & -\frac{c}{\rho Z} & 0 & -\frac{c}{\rho} & -\frac{s}{\rho} & 1
\end{array}\right]
$$

where $c=\cos \theta$ and $s=\sin \theta$.

$\widehat{L_{s_{p}}}+$ is the pseudo-inverse of $L_{s_{p}}$ evaluated with $\hat{Z}$. The image error is $e_{p}=s_{p} \ominus s_{p}^{\star}$ where $\ominus$ is the modulo $2 \pi$ subtraction for the angular component.

Remark: a degeneracy occurs for the polar representation when a point is at the origin. In order to overcome this singularity, the coordinates $(\rho, \rho \theta)$ can be used [13].

With this parametrization, the trajectories of the visual features are straight lines ( $n=3$ and the depth $Z$ known) in the polar plane but obviously not in the Cartesian plane. As can be seen on the interaction matrix (9), the rotation around the optical axis $Z$ is invariant to $\rho$ (first row, last column) and directly proportional to $\theta$ (last row, last column). Furthermore, the translation $T_{z}$ is invariant to $\theta$. This explains why the behavior obtained in the case of pure rotation around the optical axis is satisfactory. The camera movement is rotational only. On the other hand, translations along the $X$ and $Y$ axes require unnecessary displacements of the camera. 
To take advantage of the benefits of each controller, a switching control strategy was developed.

\section{SWITCHING CONTROLLER FOR IBVS}

The switching control structure (Fig 1) makes it possible to commute from the $I B V S_{C}$ controller to $I B V S_{P}$ controller, and vice versa. The decision maker provides the switching signal according to output information $s_{c}$ and criteria.

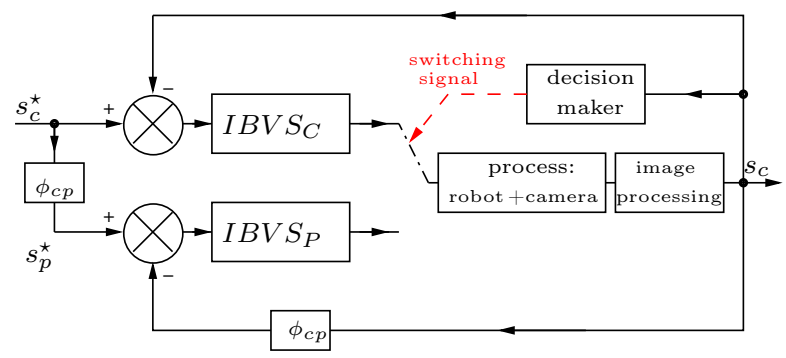

Fig. 1. Switching Control Structure

\section{A. Control law design}

The aim of the proposed controller is to be able to switch between the two controllers in order to minimize or avoid unnecessary displacements of the camera. It goes without saying that if the movement required is a pure rotation around the optical axis, the controller chosen should be the $I B V S_{P}$ controller. Similarly, if the movement is a pure translation, the decision maker should choose the $I B V S_{C}$ controller. The problem is now to determine at which instant the controller should switch from one controller to the other for any movement. Since 2D errors in Cartesian and polar coordinates have not the same scale, they cannot be compared. The information common to both controllers can only be $3 \mathrm{D}$ information, namely the movement of the camera. The suitable controller should then guarantee that the desired position is reached from the current position while minimizing the movement of the camera. To determine the controller best suited to perform the displacement, a prediction of the camera displacement to be done until convergence is carried out in parallel with the two controllers.

To perform the prediction of the camera displacement, a first step is the prediction of visual features [1]. The prediction model is based on the dynamic equation (2) (resp. (7)) in a discrete-time version. With a classical first-order discretization scheme and considering $Z$ to be approximated to $\hat{Z}$, the dynamic equations are written as:

$$
\begin{aligned}
& s_{c}(k+1)=s_{c}(k)+T_{e} \widehat{L_{s_{c}(k)}} \tau(k) \\
& s_{p}(k+1)=s_{p}(k)+T_{e} \widehat{L_{s_{p}(k)}} \tau(k)
\end{aligned}
$$

where $T_{e}$ is the sampling period.

Remark: Based on the expression of the interaction matrix, the prediction model has a local domain of validity. However, in practice, we encountered no problem of validity even for configurations that require large displacements.
Considering that one can roughly approximate the camera movement to the quantity $T_{e} \sqrt{T_{x}^{2}+T_{y}^{2}+T_{z}^{2}}$ at each iteration, we can evaluate a view of the camera displacement required up to the desired position. The prediction procedure is given below for Cartesian coordinates. It is the same for polar coordinates: the subscript ' $c$ ' is changed to ' $p$ '.

\section{Prediction procedure:}

1) At the current instant $k, i=0$;

2) Measure $s_{c}(k+i)$ thanks to the image processing,

3) Update $e_{c}(k+i)=s_{c}(k+i)-s_{c}^{\star}$ and $\widehat{L_{s_{c}}(k+i)}$,

4) Compute $\tau(k+i)=-\lambda \widehat{L_{s_{c}(k+i)}} e_{c}(k+i)$,

5) Compute $d_{c}(k+i)=T_{e} \sqrt{T_{x(k+i)}^{2}+T_{y(k+i)}^{2}+T_{z(k+i)}^{2}}$,

6) Predict $s_{c}(k+i+1)=s_{c}(k+i)+T_{e} \widehat{L_{s_{c}}(k+i)} \tau(k+i)$

7) If $e_{c}(k+i+1)=s_{c}(k+i+1)-s_{c}^{\star}<\beta_{c}$ then STOP else $i=i+1$ and go to step 4 .

$\beta_{c}$ is a small given constant and the corresponding time of convergence is noted $\left(k+f_{c}\right)$ where $f_{c}$ is the number of sampling periods needed to achieve $e_{c}<\beta_{c}$. The prediction procedure is repeated at each iteration after new measurements.

Therefore, we define the following two criteria:

$$
J_{c}=\sum_{j=k}^{k+f_{c}} d_{c}(j),
$$

for the $I B V S_{C}$ controller and in the same way, for the $I B V S_{P}$ controller:

$$
J_{p}=\sum_{j=k}^{k+f_{p}} d_{p}(j) .
$$

The decision maker should choose the $I B V S_{P}$ controller when $J_{c}>J_{p}$, and vice versa. Nevertheless, when the 2D errors are very small, the predicted $3 \mathrm{D}$ trajectories are very close for both controllers and consequently $J_{c} \cong J_{p}$. In order to avoid multiple switches of the controller, a hysteresis comparator has been implemented (see Fig. 2). If the $I B V S_{C}$ (resp. $I B V S_{P}$ ) controller was used at the previous instant, the $I B V S_{P}$ (resp. $I B V S_{C}$ ) controller will be used at the current time if and only if:

$$
J_{p}-J_{c}>T^{-} \quad\left(\operatorname{resp} . J_{p}-J_{c}<T^{+}\right)
$$

The parameter $T$ is set by simulation.

Finally, the decision maker which provides the switching signal is depicted on Figure 2. The prediction procedure and the decision making are repeated at each new sampling instant.

\section{B. Visibility constraint}

In the IBVS strategy, it is essential that the target remains in the camera field of view otherwise the feedback is no longer performed. This requirement is known as the visibility constraint. Due to the decoupling of the image errors, the $I B V S_{C}$ controller provides almost straight lines in the image plane (i.e. in the $u v$ plane) if $Z$ is estimated. The visibility of the target is then implicitly ensured. The problem of the 


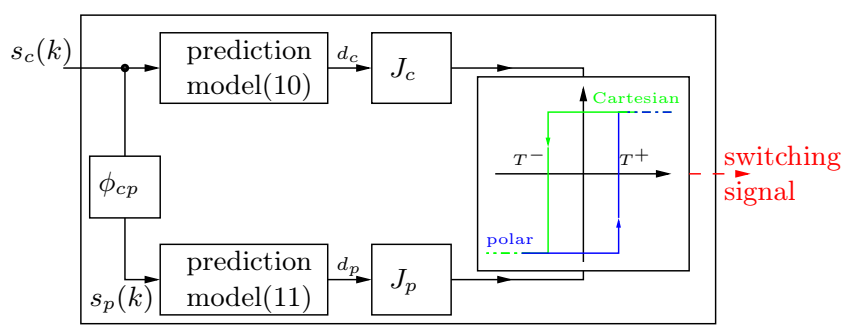

Fig. 2. Decision maker structure

target visibility can appear when the $I B V S_{P}$ controller is used. Indeed, it provides straight lines in the $\rho \theta$ plane thus resulting in circular paths in the $u v$ plane (Fig. 3). When the predicted visual features $s_{p}(k+1)$ and/or $s_{p}(k+2)$ are close to the safety zone defined in the image, the decision maker selects the $I B V S_{C}$ controller and keeps it during $N$ iterations. Once the $N$ iterations past, the standard procedure of prediction is restarted. As we will see later, $N$ equal to 10 will be sufficient in practice.

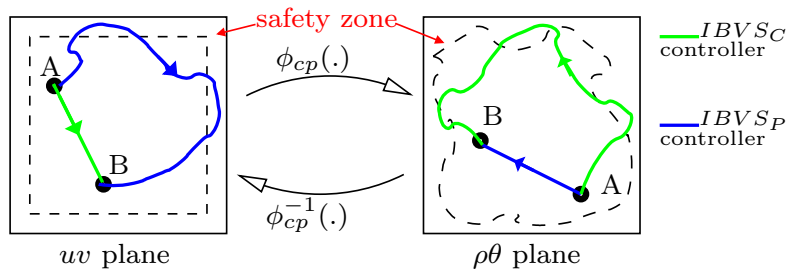

Fig. 3. Visibility constraints

\section{Stability analysis}

The stability of the closed-loop system with the classical feedback (5) or (8) has been largely studied. The local asymptotic stability is proved in both cases [2] under the assumption that the matrix $L_{s} \widehat{L}_{s}^{+}$is positive definite $(n=3)$ or positive semi-definite $\left(n>3\right.$. The matrix $L_{S} \widehat{L}_{s}^{+}$is not full rank and local minima exist). Stability of the switching system can be difficult to prove especially under an arbitrary switching signal [14]. This generally requires the stability of the controllers individually and also involves finding a Lyapunov function that is common to the two controllers.

Proposition 1: The closed-loop switching system (Fig.1) is asymptotically stable for any arbitrary switching signal within a sufficiently small neighborhood of the origin.

Sketch of Proof: Firstly, a necessary condition is, above all, that each closed-loop subsystem with the $I B V S_{C}$ controller (resp. with the $I B V S_{P}$ controller) is individually stable. Then we have to find a candidate Lyapunov function common to both subsystems. If such a function exists then the closedloop system will be stable under any arbitrary switching signal. A mapping $g$ can be defined between the image error in Cartesian coordinates and the image error in polar coordinates:

$$
\begin{aligned}
& g: \mathbb{R}^{2 * n} \rightarrow \mathbb{R}^{2 * n} \\
& e_{c} \mapsto e_{p}=g\left(e_{c}\right)=\phi_{c p}\left(s_{c}\right)-\phi_{c p}\left(s_{c}^{\star}\right), \quad g(0)=0 .
\end{aligned}
$$

Let us consider that $V_{p}$ is a common candidate function:

$$
V_{p}=\frac{1}{2} e_{p}^{T} e_{p}
$$

It is easy to show that $V_{p}=0$ if and only if $e_{p}=0$ or $e_{c}=0$ and $V_{p}=1 / 2\left\|e_{p}\right\|^{2}=1 / 2\left\|g\left(e_{c}\right)\right\|^{2}>0 . V_{p}$ is known to be a decreasing function for the $I B V S_{P}$ controller [2]. We have to prove that it is also a decreasing function for the $I B V S_{C}$ controller. In the neighborhood of the origin $e_{c}=0$, the mapping $g$ is injective and a Taylor expansion about $e_{c}=$ 0 gives:

$$
g\left(e_{c}\right)=g(0)+\left.\frac{d g}{d e_{c}}\right|_{e_{c}=0} e_{c}+o_{2}=G e_{c}+o_{2}
$$

where the jacobian $G=d g /\left.d e_{c}\right|_{e_{c}=0}$ is full column rank and $\mathrm{o}_{2}$ are terms of second order and higher. Combining (16) and (17), we get:

$$
V_{p}=\frac{1}{2} e_{c}^{T} G^{T} G e_{c}+o_{2} .
$$

Considering (2) and (5), the time derivative of (18) gives:

$$
\dot{V}_{p}=-\lambda e_{c}^{T} G^{T} G L_{s_{c}} \widehat{L_{s_{c}}} e_{c}+o_{3} .
$$

The function $V_{p}$ is a decreasing function in the neighborhood of the origin for the IBVS $S_{C}$ controller if the matrix $L_{S_{C}}{\widehat{L_{S_{C}}}}^{+}$is positive definite (or semi-definite). The candidate function $V_{p}$ is therefore a Lyapunov function common to both controllers. Consequently, the closed-loop switching system is stable under any arbitrary switching signal.

\section{SIMULATIONS}

The control task consists in positioning a perspective freeflying camera with respect to a target composed of four coplanar points forming a rectangular object. The linear and angular velocities are limited to the following bounds: $25 \mathrm{~cm} / \mathrm{s}$ for the translation speed and $0.25 \mathrm{rad} / \mathrm{s}$ for the rotation speed. The sampling period $T_{e}$ is $40 \mathrm{~ms}$ which corresponds to a usual camera ( 25 frames per second). To make the simulations more realistic, modeling errors of $20 \%$ are considered in the intrinsic camera parameters of the prediction models (10) and (11). Measurement noises are added to the outputs to simulate errors in the image processing.

\section{A. Comparative study with classical controllers}

In this section, the switching controller, noted $I B V S_{S}$, is compared with respect to the $I B V S_{C}$ controller and the $I B V S_{P}$ controller. We consider that the depth is constant and equal to the depth at the desired position $Z^{\star}$. The following color code is used for the above simulations. For the image plane and the $3 \mathrm{D}$ plane, trajectories are plotted:

- in green for the $I B V S_{C}$ controller;

- in blue for the $I B V S_{P}$ controller;

- in red for the $I B V S_{S}$ controller.

The switching signal is plotted in magenta on the control figure. When it is 0.3 (resp. -0.3), the controller $I B V S_{P}$ (resp. $I B V S_{C}$ ) is used. 
Simulation 1: case of pure rotation. We first study a pure rotation (1.4 radian) around the optical axis of the camera. All errors in the image plane converge to zero (see Fig. 4(a)). The $I B V S_{P}$ controller is used throughout the simulation as expected (see Fig. 4(b)). Indeed, the $I B V S_{C}$ controller provides straight trajectories in the image but the corresponding 3D trajectory is made up of the rotation required around the $Z$ axis and also of a translation along this axis. The latter is well-known to be useless as already mentioned in Section II. The trajectories in the image plane are circular in accordance with the 3D movement of the camera. The visual feature trajectories obtained with the switching controller are superposed to those obtained with the $I B V S_{P}$ controller.

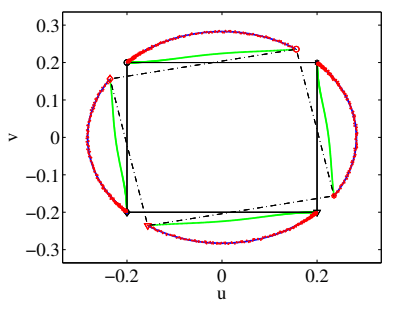

(a) Image space.

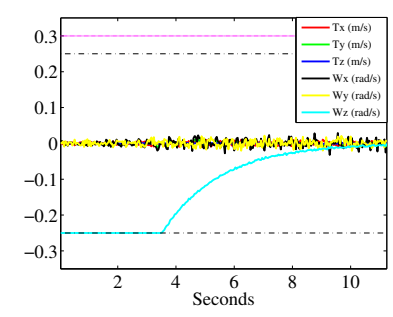

(b) control inputs
Fig. 4. Simulation 1: Case of a pure rotation

Simulation 2: case of pure translation. Translations along the $X, Y, Z$ axes are tested. As already mentioned, the $I B V S_{C}$ controller provides an optimal trajectory of the camera in $3 \mathrm{D}$ space. The switching controller selects the controller $I B V S_{C}$ throughout the simulation (see Fig. 5(c)), thus proving that the controller chosen is the best controller in this case. The trajectories in the image plane and in the 3D space tend to straight lines from the initial to the desired pose (see Fig. 5(a) and 5(b)). No additional displacement is induced.

Simulation 3: case of complex movement. The required movement of the camera is now composed of rotations and translations respectively around and along all the axes. We can see in Fig. 5(e) that the trajectories obtained with the $I B V S_{S}$ controller, in the 3D plane, are straightforward in comparison with those obtained with the $I B V S_{C}$ or the $I B V S_{P}$ controller. The camera displacement is clearly optimized: the useless displacements are minimized. The $I B V S_{C}$ is first selected for $0.5 \mathrm{sec}$ and then the $I B V S_{P}$ is chosen, etc ... (see Fig. 5(f)). The trajectories obtained with the $I B V S_{S}$ controller in the image plane satisfy the best compromise between the convergence of the visual features and the straightforward displacement of the camera (see Fig. 5(d)).

To sum up, the decision maker provides a suitable switching signal whatever the camera motion to be done. The improvements with respect to classical controllers are significant: the 2D trajectory is still satisfactory while the $3 \mathrm{D}$ trajectory is improved by avoiding unnecessary displacement.

\section{B. Comparative study with advanced controllers}

In this section, the switching controller is compared with advanced controllers to show that its performances are similar to those of advanced controllers. Among the several approaches developed to design a decoupled IBVS as already mentioned in Section I, we have selected two of them: the method using second-order minimization techniques [12], noted $I B V S_{P M J}$, and the method using image moments [5], noted $I B V S_{I M}$.

Simulation 4: $I B V S_{P M J}$ controller. In [12], two control laws were proposed. The simulation results presented here are performed by using the control law based on the pseudo-inverse of the mean of the Jacobians (PMJ). The two Jacobians are evaluated respectively at the desired position and at the current position, thus requiring the estimation of the depth $\hat{Z}$. In the case of pure rotation or translation, the simulations results of the two controllers are identical: due to the lack of place, simulation results are not shown. In the case of a displacement involving rotations and translations, the $I B V S_{S}$ controller provides the best result in both the $2 \mathrm{D}$ and 3D space (see Fig. 6(a) and 6(b)). Only one switch is needed (see Fig. 6(c)).

Simulation 5: $I B V S_{I M}$ controller. The $I B V S_{I M}$ controller is based on a combination of 6 image moments as described in [5]. For a complex movement, we can see on Fig. 6(d) that the image trajectories of the $I B V S_{I M}$ controller are more direct. The behavior of $I B V S_{S}$ controller seems to satisfy a compromise between the trajectories in the $2 \mathrm{D}$ space and in the $3 \mathrm{D}$ space.

Although designed from simple visual features (points) and using conventional interaction matrices evaluated at the desired position, the effectiveness of the switching controller is similar to the advanced controllers's one.

\section{Visibility constraint}

The challenging difficulty of visual servoing is to keep the image features in the camera field of view. In order to illustrate the efficiency of the $I B V S_{S}$ controller, visibility constraints are taken into account in the relevant case of pure rotation (1.4 radian).

Simulation 6: Considering the image size to be \pm 0.3 , a safety zone is delimited by bounds on $u$ and $v$ : $u^{-}=v^{-}=-0.25$ and $u^{+}=v^{+}=0.25$. The convergence of the visual features to the desired position is still achieved while the visibility constraints are satisfied (see Fig. 7(a)). To do this, multiple switches were needed throughout the simulation (see Fig. 7(b)). While the $I B V S_{P}$ controller seems optimal from the 3D point of view for a pure rotation around the optical axis, the image trajectories can get out of the safety zone. A switch to the $I B V S_{C}$ controller ensures quasi-straight trajectories in the image and thus implicitly ensures the satisfaction of visibility constraints. Consequently, a slight translational displacement of the 


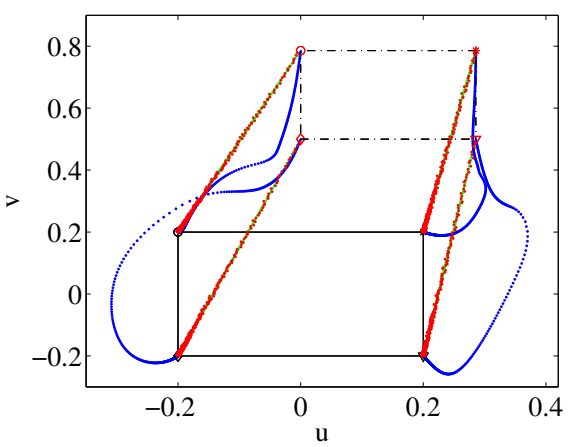

(a) Simulation 2: 2D space.

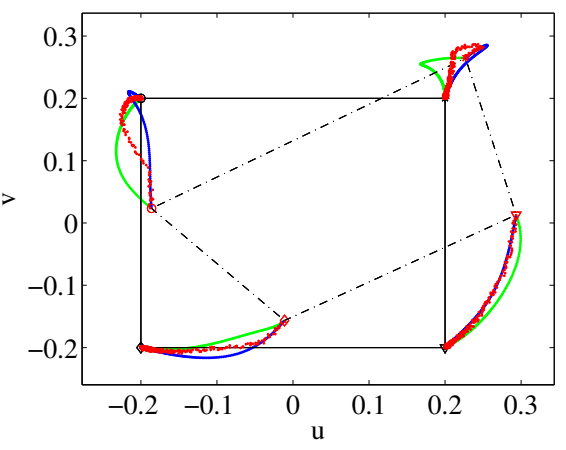

(d) Simulation 3: 2D space.

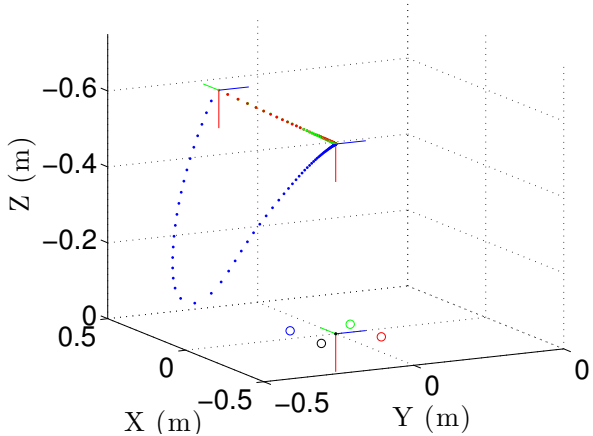

(b) Simulation 2: 3D space.

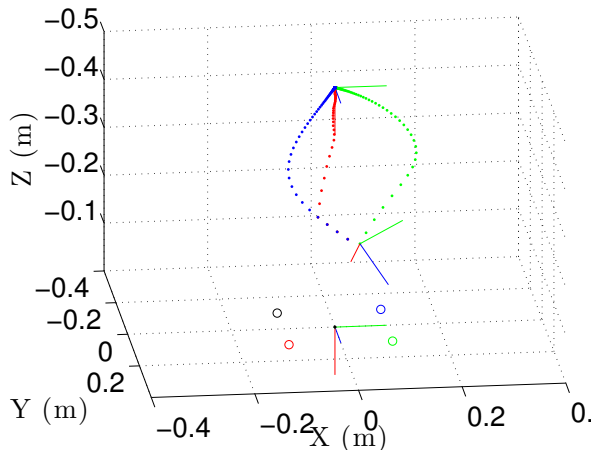

(e) Simulation 3: 3D space.

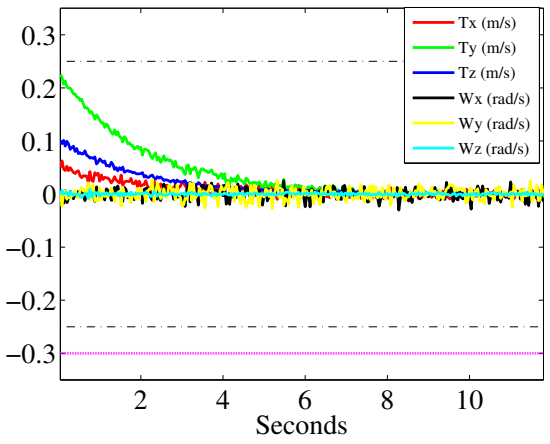

(c) Simulation 2: Control inputs.

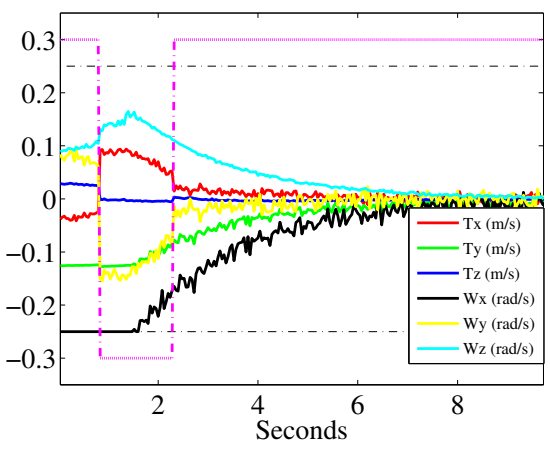

(f) Simulation 3: Control inputs.

Fig. 5. Simulation 2: case of pure translation. Simulation 3: case of complex movement

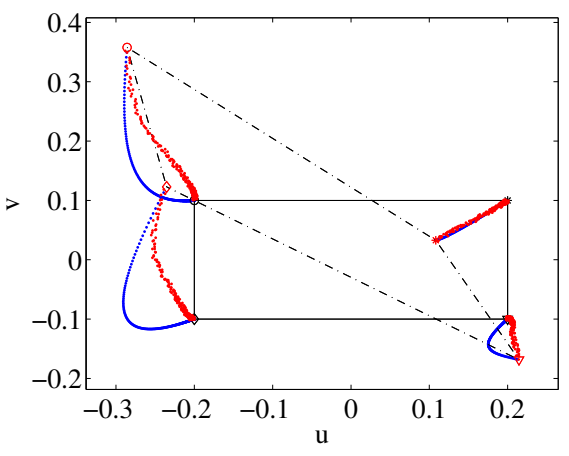

(a) Simulation 4: 2D space.

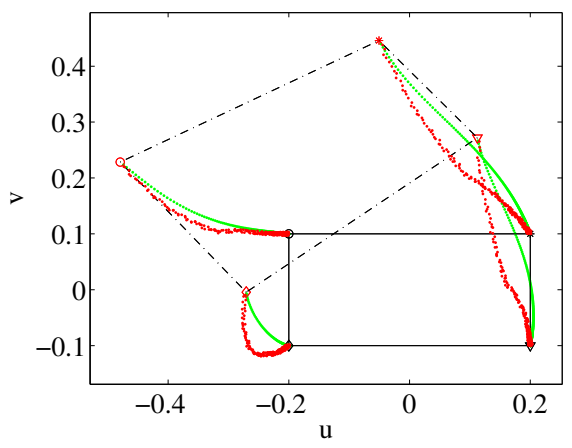

(d) Simulation 5: $2 \mathrm{D}$ space.

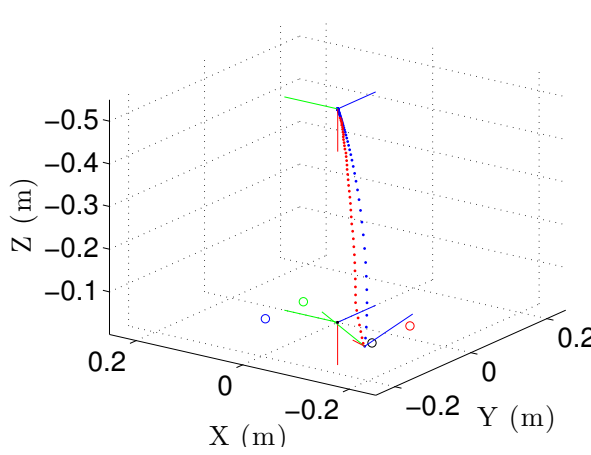

(b) Simulation 4: 3D space.

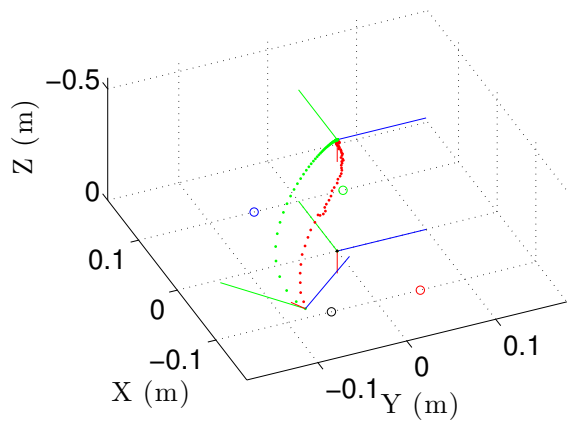

(e) Simulation 5: 3D space.

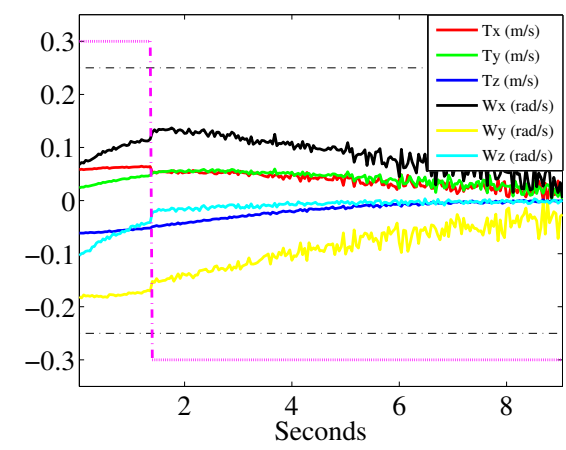

(c) Simulation 4: Control inputs.

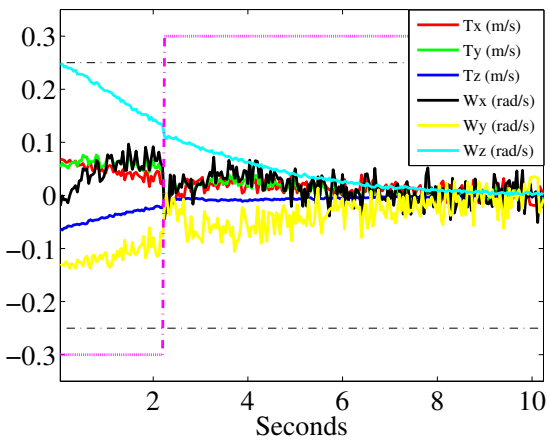

(f) Simulation 5: Control inputs.

Fig. 6. Simulation 4: $I B V S_{P M J}$ controller (in blue) vs $I B V S_{S}$ controller (in red). Simulation 5: $I B V S_{I M}$ controller (in green) vs $I B V S_{S}$ controller (in red). 


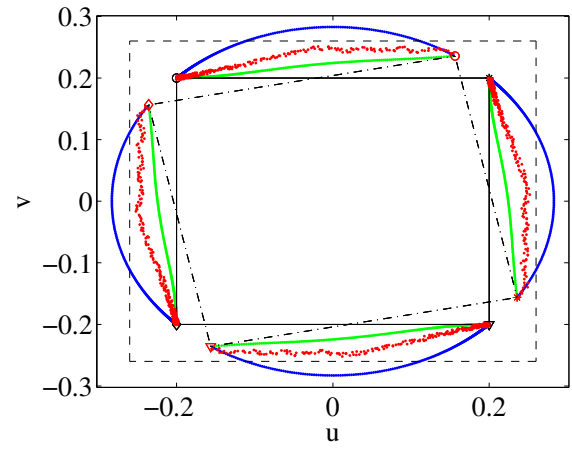

(a) Image space

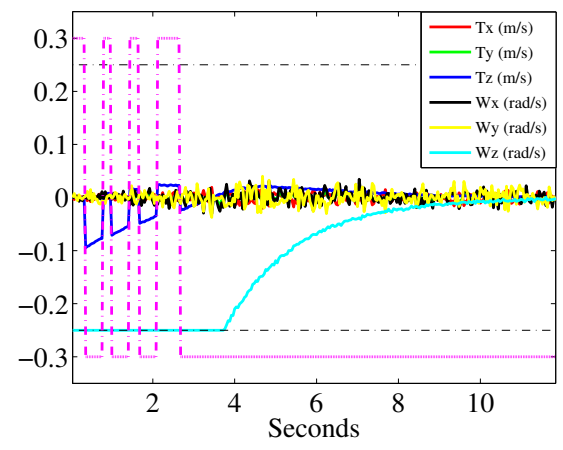

(b) Control inputs

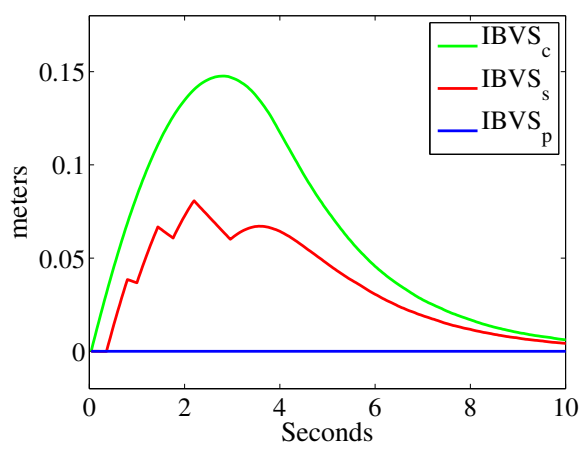

(c) displacement along $\mathrm{Z}$ axis

Fig. 7. Simulation 6: Case of a pure rotation with visibility constraints

camera along the optical axis is induced by the $I B V S_{C}$ controller. This displacement, which is essential to guarantee the feature visibility, is about $8 \mathrm{~cm}$ against $14 \mathrm{~cm}$ if only the $I B V S_{C}$ controller were used throughout the simulation (see Fig. 7(c)).

Although the control law generated by the controller $I B V S_{S}$ is not continuous, it is important to note that this control is applied as a reference input for low-level controllers servoing the joints of the robotic system. Therefore, the rapid changes are attenuated as was shown in practice in [10]. In the worst cases, it is possible to filter the computed control before sending it to the low-level controllers.

The average computational time devoted to the prediction of the camera movement for both controllers is about $20 \mathrm{~ms}$ at each iteration and can be greatly reduced (with a $\mathrm{C}$ language) for a real time application.

\section{CONCLUSIONS}

In this paper, we have proposed a switching controller for IBVS tasks. The switching controller takes advantage of the benefits of Cartesian-based IBVS and polar-based IBVS. If the tools used (Cartesian IBVS, polar IBVS, switch, predictive approach) are well known, their combination is original and new. The switching approach is an alternate approach to design a decoupled IBVS in an easy way with point visual features and no 3D information. The visual features efficiently converge to the desired position while the $3 \mathrm{D}$ displacement of the camera is minimized. The decision maker provides the switching signal according to criteria based on the predicted displacements of the camera for the two controllers, without 3D reconstruction. The stability of the closed-loop switching system is locally proved for any arbitrary switching signal. The enhancement of the IBVS performances is illustrated through the simulation results. The robustness of the switching controller is checked in the presence of modeling errors and measurement noise. The handling of visibility constraints can be improved by introducing a potential function as in [6]. The next step will be the implementation of the switching controller on a $6 \mathrm{ddl}$ arm manipulator.

\section{REFERENCES}

[1] G. Allibert, E. Courtial, F. Chaumette, Predictive Control for Constrained Image-Based Visual Servoing, IEEE Trans. on Robotics, vol. 26(5), pp 933-939, October 2010.

[2] F. Chaumette, and S. Hutchinson, Visual Servo Control, Part I: Basic Approaches, IEEE Robotics and Automation Magazine, vol. 13(4), pp 82-90, December 2006.

[3] F. Chaumette, and S. Hutchinson, Visual Servo Control, Part II: Advanced Approaches, IEEE Robotics and Automation Magazine, vol. 14(1), pp 109-118, March 2007.

[4] F. Chaumette, Potential problems of stability and convergence in image-based and position-based visual servoing, The Confluence of Vision and Control, LNCIS Series, No 237, pp 66-78, Springer-Verlag, 1998.

[5] F. Chaumette, Image moments: a general and useful set of features for visual servoing, IEEE Trans. on Robotics, vol. 20(4), pp 713-723, August 2004.

[6] P. Corke and S. Hutchinson, A new partitioned approach to imagebased visual servo control, IEEE Trans. Robot. Automat., vol. 17, pp. 507-515, August 2001.

[7] P. Corke, and F. Spindler, and F. Chaumette, Combining Cartesian and cylindrical coordinates in IBVS, IEEE Int. Conf. on Intelligent Robots and Systems, pp 5962-5967, St Louis, USA, October 2009.

[8] K. Deguchi, Optimal motion control for image-based visual servoing by decoupling translation and rotation, IEEE Int. Conf. on Intelligent Robots and Systems, pp 705-711, Victoria, Canada, October 1998.

[9] L. Deng, F. Janabi-Sharifi, and W. Wilson, Hybrid motion control and planning strategies for visual servoing, IEEE Transactions on Industrial Electronics, vol. 52(4), pp 1024-1040, August 2005.

[10] N.R. Gans and S.A. Hutchinson, Stable Visual Servoing Through Hybrid Switched-System Control, IEEE Transactions on Robotics, vol. 23(3), pp 530-540, June 2007.

[11] E. Malis, F. Chaumette and S. Boudet, 2 1/2 D Visual Servoing, IEEE Trans. on Robotics and Automation, vol. 15(2), pp 238-250, April 1999.

[12] E. Malis, Improving vision-based control using efficient second-order minimization techniques, IEEE Int. Conf. on Robotics and Automation, New Orleans, USA, April 2004.

[13] M. Iwatsuki and N. Okiyama, A new formulation of visual servoing based on cylindrical coordinate system with shiftable origin, in IEEE/RSJ Int. Conf. Intelligent Robots and System, vol. 1, pp 354$359,2002$.

[14] D. Liberzon, Switching in Systems and Control, Birkhauser, ISBN 08176-4297-8, 2003. 